\title{
VALOR RAZONABLE Y LA CALIDAD DE INFORMACIÓN REVELADA: EFECTOS DE LA APLICACIÓN ANTICIPADA DE LA NIIF 13 EN CHILE*
}

\section{FAIR VALUE AND THE QUALITY OF INFORMATION DISCLOSURE: EFFECTS OF THE EARLY ADOPTION OF IFRS 13 IN CHILE}

\author{
Hanns de la Fuente Mella ${ }^{1}$, Berta Silva Palavecinos ${ }^{2}$, Ricardo Campos Espinoza ${ }^{3}$ \\ y David Cademartori Rosso ${ }^{4}$
}

\section{RESUMEN}

El mercado de capitales chileno se caracteriza por alta concentración de la propiedad y por la participación de inversionistas institucionales como las Administradoras de fondos de Pensiones (AFP). En Chile, las IFRS se adoptan a partir del año 2009. Desde la emisión de la IFRS 13, en 2011, su aplicación ha sido voluntaria, y sólo obligatoria a partir del 2013.

El propósito de este estudio es medir los efectos de la adopción anticipada del uso del valor razonable en la transparencia del mercado de capitales chileno y en la disminución de asimetría de información.

Como proxy de la asimetría de información se trabajará con el spread (bid-ask) con datos de acciones intra-diarios de la Bolsa de Comercio de Santiago para el período 2007-2012.

El estudio muestra que la aplicación anticipada de la IFRS 13 no ha producido efecto significativo en la asimetría de información, se aprecia además que la aplicación de la norma no ha producido el efecto esperado, es decir, a menor nivel de jerarquía del valor razonable menor es la asimetría de información.

Palabras claves: Asimetría de información; jerarquía de valor razonable; spread (bid-ask).

Recepción: 14/09/2015. Aprobación: 27/01/2016.

\section{ABSTRACT}

The Chilean capital market is characterized by high concentration of ownership and the participation of institutional investors such as Pension Funds (AFP). In Chile, the IFRS are adopted from the year 2009. Since the issuance of IFRS 13, in 2011, its application has been voluntary, and only mandatory from 2013.

\footnotetext{
* Los autores agradecen el apoyo financiero de la Pontificia Universidad Católica de Valparaíso a través del Proyecto de investigación VRIEA-PUCV 37.355/ 2014 y la colaboración recibida de la Bolsa de Comercio de Santiago por los datos proporcionados para este artículo.

1 Pontificia Universidad Católica de Valparaíso, Facultad de Ciencias Económicas y Administrativas, Escuela de Comercio, Valparaíso, Chile. E-mail: hanns.delafuente@ucv.cl

2 Pontificia Universidad Católica de Valparaíso, Facultad de Ciencias Económicas y Administrativas, Escuela de Comercio, Valparaíso, Chile. E-mail: bsilva@ucv.cl

3 Pontificia Universidad Católica de Valparaíso, Facultad de Ciencias Económicas y Administrativas, Escuela de Comercio, Valparaíso, Chile. E-mail: ricardo.campos.e@ucv.cl

4 Pontificia Universidad Católica de Valparaíso, Facultad de Ciencias Económicas y Administrativas, Escuela de Comercio, Valparaíso, Chile. E-mail: dcademar@ucv.cl
} 
The purpose of this study is to measure the impact of the early adoption of the use of fair value in the transparency of the Chilean capital market and the reduction of information asymmetry.

As a proxy of information asymmetry will be used the spread (bid-ask) with intra-day data of the Santiago Stock Exchange for the period 2007-2012.

The study shows that the early adoption of IFRS 13 has not produced significant effect on the asymmetry of information, it is further appreciated that the application of the standard has not produced the expected effect, that is, at lower level of fair value hierarchy less asymmetry of information.

Keywords: Asymmetry of information; fair value hierarchy; spread (bid-ask).

\section{INTRODUCCIÓN Y REVISIÓN DE LA LITERATURA}

Desde la Teoría Económica se establece que la correcta asignación de recursos supone un mercado eficiente y ello implica entre otros aspectos, que todos los actores del mismo dispongan de información suficiente, relevante y fidedigna. La disminución de la asimetría de información es muy importante, por una parte se beneficia a los inversionistas otorgando igualdad de oportunidades para invertir o desinvertir; especialmente, beneficia a los accionistas minoritarios, entre los cuales se encuentran los inversionistas institucionales; y por otra, facilita el acceso a fuentes de financiamiento ya que disminuye el riesgo y por lo tanto disminuye el costo de capital permitiendo aceptar proyectos que en caso contrario serían rechazados.

El spread (bid-ask) de las acciones ha sido considerado por muchos autores un proxy para medir la asimetría de información (Liao, Kang, Morris \& Tang, 2013; Muller, Riedl \& Sellhorn, 2011; Kanagaretnam, Lobo \& Whalen, 2007; Dennis \& Weston, 2001; Healy \& Palepu, 2001; Sarin, Shastri \& Shastri, 1996; Kim \& Verrecchia, 1994; Venkatesh \& Chiang, 1986; Copeland \& Galai, 1983) por ejemplo, los traders amplían el bid-ask para cubrirse de pérdidas por posibles asimetrías de información (Glosten \& Harris, 1998).

La globalización y la necesidad de mejorar la eficiencia de los mercados ha hecho necesario tener información que refleje lo más fielmente posible el resultado de la gestión en los negocios. En el modelo de valor razonable la medición se realiza en base a datos que pueden ser observables o no. Datos observables son aquellos elaborados en base a información disponible en el mercado activo y que refleja los supuestos que los participantes usarían para fijar precio al activo o pasivo. Datos no observables, son aquellos para los cuales no hay información de mercado disponible y que son elaborados en base a la mejor información disponible acerca de los supuestos que los participantes usarían para fijar el precio al activo o pasivo. Es así como los datos de Nivel 1, corresponden a precios cotizados (sin ajustar) en mercados activos para activos y pasivos idénticos, a los cuales la entidad puede tener acceso en la fecha de medición. Los datos de Nivel 2, son distintos de los precios cotizados incluidos dentro del Nivel 1, que son observable para el activo o el pasivo, ya sea directamente (bajo forma de precio), sea indirectamente (determinado a partir de precio). Los datos de Nivel 3 son concernientes al activo o al pasivo que no están basados sobre datos observables del mercado. Los datos no observables reflejarán los supuestos que los participantes de mercado utilizarían al fijar el precio del activo o pasivo, incluyendo supuestos sobre el riesgo (IASB, 2011). La jerarquía de valor razonable da la mayor importancia al Nivel 1 y la menor importancia de los datos no observables al nivel 3 (IASB, 2011).

La demanda de información financiera y el requerimiento de su divulgación, se deben a la necesidad de disminuir la asimetría de la información derivado del conflicto de agencias entre los administradores y los inversionistas que no participan en la gestión y dirección de la empresa (Healy \& Palepu, 2001), o de la existencia de información privilegiada por parte de los accionistas controladores, solos o en colusión con los administradores de la empresa, ambas situaciones perjudican a los accionistas minoritarios (Maug, 2002). Las empresas que adoptan pobres prácticas de transparencia y revelación de información experimentarán graves asimetrías (Chen, Chung, Lee \& Liao, 2007).

El Marco Conceptual de 2010 tiene por objeto proporcionar información financiera sobre la entidad que informa, que sea útil para los inversionistas y acreedores, existentes o potenciales. Hay diversos estudios, por ejemplo, Barth, Landsman, Lang \& Williams (2012), Clarkson, Hanna, Richardson \& Thompson (2011), que analizan si la adopción de IFRS contribuye a proporcionar 
información financiera más transparente y comparable.

Munteanu (2011) en una extensa revisión a la literatura concluye que existe una disminución del costo de capital en aquellas empresas que han adoptado IFRS en forma seria y voluntaria, también constata que en el caso de adopción obligatoria de la norma, existe disminución de la calidad de la información, aun cuando no sea significativa. Armstrong, Barth, Jagolinger \& Riedl (2010) analizan el impacto de 16 eventos asociados a la adopción de IFRS en Europa y concluyen que la adopción produce una disminución en el costo de capital.

La calidad de los gobiernos corporativos, relacionada significativamente con la asimetría de información, en algunos casos es medida en base a la información que revelan las empresas en forma pública, en la literatura se encuentra el índice de revelación de información (DIS, por Disclosure Index) propuesto por Haat, Mahenthiran, Rahman \& Hamid (2006), que está compuesto por 17 ítems que recogen aspectos de la calidad de la información revelada. Otra propuesta es el índice construido por Botosan (1997), que recoge 138 ítems agrupados en 18 grupos, abarcando un espectro más amplio de características de la información que las empresas divulgan. Kanagaretnam et al. (2007) demuestran que mientras mejor es la calidad de los gobiernos corporativos menor es la asimetría de información, relacionando el spread con variables como los directores independientes y otras características del directorio, usando como variable de control entre otras, el tamaño de la empresa.

Adicionalmente, Liao et al. (2013) encuentran que todos los niveles de valor razonable están positiva y significativamente asociados con la asimetría de información, siendo mayor para el nivel 3 y menor para el nivel 1. Por otra parte, otros autores muestran que la introducción de las IFRS no ha contribuido a mejorar en forma significativa la calidad de la información ( $\mathrm{Mu}-$ ller et al., 2011). La aplicación de la IFRS 13 no tuvo un efecto significativo en la crisis financiera (Barth y Landsman, 2010).

Algunos estudios mencionan que en mercados emergentes, el uso del valor razonable en la contabilidad no genera los beneficios esperados de una mayor transparencia $(\mathrm{He}$, Wong \& Young, 2012). En Chile, el organismo regulador de las sociedades que cotizan en bolsa indicó que dichas compañías debían adoptar las IFRS a partir del año 2009. Aunque a esa fecha no existía la IFRS 13, las compañías chilenas la aplicaron basándose en el FAS 157.
Respecto a la participación de los inversionistas institucionales en la propiedad de las empresas, se puede destacar que la OCDE (2011, pág. 3) considera que el rol de estos es más crítico en América Latina que en otras partes del mundo. Esta importancia deriva de la característica de alta concentración de la propiedad que prevalece en los mercados latinoamericanos y la existencia del riesgo de perjuicio para los accionistas minoritarios.

El spread aumenta mientras mayor es la propiedad que tienen los administradores y disminuye cuando la propiedad es de otros inversionistas, especialmente los institucionales y cuando existen directores independientes (Bakhshi, Bazrafshan, Rezaei \& Fereidouni, 2011). La propiedad que concentran los cinco primeros accionistas en el mercado chileno es muy alta comparada con otros mercados (Rubin, 2007). Los cinco primeros accionistas de las empresas que componen el IPSA al año 2013 explican el $67 \%$ de la propiedad.

Dennis y Weston (2001) encuentran que la relación entre propiedad institucional y spread es negativa y significativa, al contrario de Sarin et al. (1996) que encuentran una relación significativa pero positiva Florou \& Pope (2012) analizan los efectos de la adopción de las IFRS y concluyen que la mejor calidad de los estados financieros ha afectado la conducta de los inversionistas institucionales.

Por último, hay evidencias de que la incorporación de una empresa a un índice bursátil disminuye el spread, disminuye la asimetría de información (Erwin \& Miller, 1998; Assidi \& Omri, 2012). De esta manera considerar si las empresas estudiadas pertenecen al IPSA (Índice de Precios Selectivo de Acciones) en un determinado año, servirá para controlar este efecto en el modelo propuesto.

El objetivo fundamental del estudio es analizar cómo afecta al spread la aplicación del valor razonable de los activos y pasivos financieros. Para lograr el objetivo mencionado es necesario medir la calidad de los gobiernos corporativos a través de la calidad de la información revelada, mediante índices de revelación y medir el uso del valor razonable. Para lo primero se usará un instrumento desarrollado por Haat et al. (2006). Adicionalmente, se usará la metodología sugerida por Botosan (1997). Para lo segundo, se trabajará con los distintos niveles revelados de determinación del valor razonable.

De acuerdo a lo señalado en la revisión de la literatura, las hipótesis que se plantean en la presente investigación son: 
H1: la aplicación anticipada de la IFRS 13 genera menos asimetría de información.

H2: La asimetría de información es una función creciente en relación al nivel usado para la valoración de activos y pasivos financieros, es decir, a mayor nivel de valor razonable mayor asimetría.

\section{MATERIALES Y MÉTODOS}

El análisis empírico se realiza para una muestra de 65 empresas que cotizan en la Bolsa de Comercio de Santiago de Chile La mayoría de las empresas seleccionadas han tenido presencia continua en el IPSA durante el período 20072012 y el total de la muestra representa al $1^{\circ}$ de abril de 2014 ell 131\% del total del patrimonio bursátil de las empresas de este índice ${ }^{5}$.

Los datos para calcular el spread se han obtenido desde la Bolsa de Comercio de Santiago ${ }^{6}$ y los datos relativos a las compañías desde las memorias corporativas publicadas por la Superintendencia de Valores y Seguros (SVS). Todos los datos son anuales y sintetizan sus operaciones corporativas.

El 1 modelo general incluye como variable endógena el spread, correspondiente a la serie de precios de compra y venta de las acciones de las empresas de la muestra. El spread se ha medido de dos maneras: (i) como el promedio anual del logaritmo natural de las diferencias entre ambos precios (Spread_Logaritmo), (ii) como el promedio anual de la diferencia de ambos precios dividido por su valor medio (Spread_Medio). Por otro lado, las variables exógenas del modelo son las siguientes: (a) tres variables dicotómicas, una que indica si la empresa ha adoptado las disposiciones de la IFRS 13 en la medición y revelación de información de los activos y pasivos financieros $(V R)$; otra que muestra si la empresa ha adoptado las IFRS (IFRS), y una última que indica si la empresa pertenece al IPSA en el periodo de estudio (IPSA); (b) 5 variables de control: el nivel de endeudamiento corporativo (Leverage), el tamaño del activo de las empresas (LN_Activos), el porcentaje de presencia bursátil de las empresas de la muestra ( $P \_$Bursatil), la participación de los inversionistas institucionales ${ }^{7}$ en la propiedad de las empresas de la muestra que les da derecho a tener un director independiente (Prop_Inv Inst_AFP), y el porcentaje de propiedad de los 5 principales accionistas (Prop_5_Accionistas); (c) 6 variables que indican el tipo de datos de entrada utilizados para medir el valor razonable de los activos y pasivos financieros que poseen las empresas es (N1_AF; N1_PF; N2_AF; N2_PF; N3_AF; N3_PF), siendo el primero de los niveles, el que proporciona más información observable en el mercado; y por último, (d) dos variables de la calidad de los gobiernos corporativos, medida a través de dos indicadores: DIS y Botosan. Para DIS se utiliza su valor obtenido del cálculo de los 17 ítems (DIS), y para Botosan, se usa el valor obtenido del cálculo de los 138 ítems (Botosan).

El modelo general en su forma funcional queda especificado por la siguiente ecuación:

Spread $_{\mathrm{i}}=\beta_{0}+\beta_{1}{ }^{*} \mathrm{VR}_{\mathrm{i}}+\beta_{2}{ }^{*} \operatorname{IFRS}_{\mathrm{i}}+\beta_{3}{ }^{*} \operatorname{IPSA}_{\mathrm{i}}+\beta_{4}{ }^{*}$ Leverage $_{\mathrm{i}}+\beta_{5}{ }^{*} \operatorname{Ln}_{-}$Activos $_{\mathrm{i}}+\beta_{6}{ }^{*} \mathrm{P}_{-}$Bursatil $_{\mathrm{i}}+\beta_{7}{ }^{*}$ Prop $_{-}$ Inv_Inst_AFP ${ }_{i}+\beta_{8}{ }^{*}$ Prop_5_Accionistas $_{i}+\beta_{9}{ }^{*} \mathrm{~N} 1 \_A F_{i}+\beta_{10}{ }^{*} \mathrm{~N} 1 \_P F_{i}+\beta_{11}{ }^{*} \mathrm{~N} 2 \_A F_{i}+\beta_{12}{ }^{*} \mathrm{~N} 2{ }_{2} \mathrm{PF}_{\mathrm{i}}+$ $\beta_{13}{ }^{*} \mathrm{~N} 3 \_\mathrm{AF}_{\mathrm{i}}+\beta_{14}{ }^{*} \mathrm{~N} 3 \mathrm{PF}_{\mathrm{i}}+\beta_{15}{ }^{*} \mathrm{DIS}{ }_{\mathrm{i}}+\beta_{16}{ }^{*}$ Botosan $_{\mathrm{i}}+\mu_{\mathrm{i}}$

(ecuación 1)

En la Tabla I es posible observar los principales datos de análisis exploratorio de las variables continuas del modelo. Se puede apreciar que las magnitudes de las variables resultan bastante heterogéneas en función de su naturaleza. De la muestra, en el año 2009 la mitad de las compañías adoptaron el uso de las IFRS y a partir de 2010 la totalidad estaba en régimen full IFRS. Por otra parte, desde el año de emisión de la IFRS 13 (en 2011) el 56\% de las empresas revelaron información referida a la valorización de activos y pasivos financieros bajo dicha norma, adoptándola de manera anticipada, porcentaje que se mantuvo en 2012. Se puede destacar que

5 Al 1-4-2014 la capitalización total de las acciones que participan en el IPSA asciende a MM\$104,063,246, mientras que el valor de la capitalización bursátil de las acciones de la muestra es de MM\$117,716,729

6 Se consideran sólo las transacciones realizadas entre las 11:00-14:00, objeto evitar el sesgo causado por las transacciones realizadas al inicio y término del día que pueden quedar pendientes para el día siguiente (Gjerde, Mahenthiran \& Cademartori, 2013).

7 Según la Superintendencia de Valores y Seguros de Chile los inversionistas institucionales son organizaciones que operan grandes volúmenes de activos: bancos, sociedades financieras, compañías de seguro y nacionales de reaseguro, administradoras de fondos de pensiones (AFP) y administradoras de fondos autorizados por ley. 
antes de la emisión de IFRS 13, las compañías aplicaron las directrices de valor razonable (33\% en 2009 y 53\% en 2010), seguramente aplicando FAS 157 para cumplir con normas de inversionistas de EUA.

También se encuentran evidencias en la muestra de una relación positiva entre la participación de las acciones corporativas en el IPSA y la adopción de IFRS y entre la participación en el IPSA y la aplicación de la IFRS 13. El 6\% de los casos registrados (14 sobre 246) no participaban del IPSA y no adoptaron IFRS, en cambio el 57\% (139 sobre 246) participaba del IPSA y también adoptó IFRS (entre 2009 y 2012). En la misma dirección, de todos los casos de la muestra el 36\% aplicó e informó valor razonable y no participó en el IPSA, versus el $64 \%$ de los casos aplicó dicha norma mientras la acción estuvo incluida en el IPSA.

Tabla I. Estadísticos descriptivos de las variables usadas en los modelos.

\begin{tabular}{|l|l|l|l|l|l|l|}
\hline Variable & Mínimo & Máximo & Media & $\begin{array}{l}\text { Desviación } \\
\text { Estándar }\end{array}$ & Asimetría & Curtosis \\
\hline Spread_Medio & 0.00137946 & 0.21181517 & 0.01811734 & 0.02179883 & 3.88891344 & 23.1773427 \\
\hline Spread_Logaritmo & 0.00137946 & 0.26098565 & 0.01846291 & 0.02347304 & 4.70024492 & 35.8774346 \\
\hline Botosan & 9 & 49 & 29.7886297 & 9.8261832 & 0.08945416 & -0.9841408 \\
\hline DIS & 0.3529412 & 0.7411765 & 0.57591186 & 0.07412117 & -0.19118926 & -0.04697065 \\
\hline VR & 0 & 1 & 0.27423823 & 0.4467488 & 1.01631834 & -0.97251579 \\
\hline N1_AF & 0 & 0.1717966 & 0.01578724 & 0.03341381 & 2.75547955 & 7.7479166 \\
\hline N1_PF & 0 & 0.13093872 & 0.0029318 & 0.01778247 & 6.49368098 & 42.4563204 \\
\hline N2_AF & 0 & 0.24074519 & 0.01351904 & 0.03375956 & 4.08753451 & 21.4815249 \\
\hline N2_PF & -0.00540492 & 0.29682362 & 0.01122745 & 0.03245381 & 7.17812018 & 61.5474184 \\
\hline N3_AF & 0 & 0.8307831 & 0.02480369 & 0.13565003 & 5.5730172 & 29.7447538 \\
\hline N3_PF & 0 & 0.02257224 & 0.00030569 & 0.00227188 & 9.71074919 & 95.9426847 \\
\hline
\end{tabular}

Para esta aplicación empírica se trabaja con cuatro modelos de corte transversal ${ }^{8}$ separados en dos grupos. Para el primer grupo se utiliza una variable dicotómica que indica si las empresas de la muestra han adoptado las disposiciones de la IFRS 13 en la medición y revelación de información de los activos y pasivos financieros; mientras que para el segundo grupo de modelos, se identifica y cuantifica la separación en niveles en la medición de los activos y pasivos financie- ros de cada una de las empresas de la muestra.

Para ambos grupos de modelos, se trabaja con dos variables endógenas donde cada una representa diferentes mediciones del Spread (De la Fuente, Campos, Silva \& Cademartori, 2013). Así, el primero usa como medida del spread el Spread_Logaritmo, mientras que para el segundo modelo, se usa Spread_Medio. Funcionalmente los cuatro modelos quedan especificados por las siguientes ecuaciones:

Spread_Logaritmo ${ }_{\mathrm{i}}=\beta_{0}+\beta_{1}{ }^{*} \mathrm{VR}_{\mathrm{i}}+\beta_{2}{ }^{*} \operatorname{IFRS}_{\mathrm{i}}+\beta_{3}{ }^{*} \operatorname{IPSA}_{\mathrm{i}}+\beta_{4}{ }^{*}$ Leverage $_{\mathrm{i}}+\beta_{5}{ }^{*} \operatorname{Ln}_{-}$Activos $_{\mathrm{i}}+\beta_{6}{ }^{*} \mathrm{P}_{-}$Bursatil $_{\mathrm{i}}$ $+\beta_{7}{ }^{*}$ Prop_Inv_Inst_AFP $_{\mathrm{i}}+\beta_{8}{ }^{*}$ Prop_5_Accionistas $_{\mathrm{i}}+\beta_{9}{ }^{*} \mathrm{DIS}_{\mathrm{i}}+\beta_{10}{ }^{*}$ Botosan $_{\mathrm{i}}+\mu_{\mathrm{i}}$

(ecuación 2)

Spread_Medio ${ }_{i}=\beta_{0}+\beta_{1}{ }^{*} \operatorname{VR}_{\mathrm{i}}+\beta_{2}{ }^{*} \operatorname{IFRS}_{\mathrm{i}}+\beta_{3}{ }^{*} \operatorname{IPSA}_{\mathrm{i}}+\beta_{4}{ }^{*}$ Leverage $_{\mathrm{i}}+\beta_{5}{ }^{*} \operatorname{Ln}_{-}$Activos $_{\mathrm{i}}+\beta_{6}{ }^{*} \mathrm{P}_{-}$Bursatil $_{\mathrm{i}}+$ $\beta_{7}{ }^{*}$ Prop_Inv_Inst_AFP $_{\mathrm{i}}+\beta_{8}{ }^{*}$ Prop_5_Accionistas $_{\mathrm{i}}+\beta_{9}{ }^{*}$ DIS $_{\mathrm{i}}+\beta_{10}{ }^{*}$ Botosan $_{\mathrm{i}}+\mu_{\mathrm{i}}$

(ecuación 3)

8 La combinación de empresas/ periodos es para cada una de las secciones transversales del modelo. 
Spread_Logaritmo $_{i}=\beta_{0}+\beta_{1}{ }^{*}$ IFRS $_{i}+\beta_{2}{ }^{*}$ IPSA $_{i}+\beta_{3}{ }^{*}$ Leverage $_{i}+\beta_{4}{ }^{*} \operatorname{Ln}_{-}$Activos $_{i}+\beta_{5}{ }^{*}$ P_Bursatil $_{1}+$ $\beta_{6}{ }^{*}$ Prop_Inv_Inst_AFP $+\beta_{7}{ }^{*}$ Prop_5_Accionistas $_{\mathrm{i}}+\beta_{8}{ }^{*} \mathrm{~N} 1 \_\mathrm{AF} \mathrm{F}_{\mathrm{i}}+\beta_{9}{ }^{*} \mathrm{~N} 1{ }_{-} \mathrm{PF} \mathrm{F}_{\mathrm{i}}+\beta_{10}{ }^{*} \mathrm{~N} 2{ }^{*} \mathrm{AF} \mathrm{F}_{\mathrm{i}}+\beta_{11}{ }^{*} \mathrm{~N} 2 \_\mathrm{PF}$ $+\beta_{12}{ }^{*} \mathrm{~N} 3{ }_{-} \mathrm{AF} \mathrm{i}_{\mathrm{i}}+\beta_{13}{ }^{*} \mathrm{~N} 3{ }_{-} \mathrm{PF}_{\mathrm{i}}+\beta_{14}{ }^{*} \mathrm{DIS}_{\mathrm{i}}+\beta_{15}{ }^{*}$ Botosan $_{\mathrm{i}}+\mu_{\mathrm{i}}$

(ecuación 4)

Spread_Medio ${ }_{\mathrm{i}}=\beta_{0}+\beta_{1}{ }^{*} \operatorname{IFRS}_{\mathrm{i}}+\beta_{2}{ }^{*}$ IPSA $_{\mathrm{i}}+\beta_{3}{ }^{*}$ Leverage $_{\mathrm{i}}+\beta_{4}{ }^{*} \operatorname{Ln}_{-}$Activos $_{\mathrm{i}}+\beta_{5}{ }^{*} \mathrm{P}_{-}$Bursatil $_{\mathrm{i}}+\beta_{6}{ }^{*}$ Prop $_{-}$ Inv_Inst_AFP ${ }_{\mathrm{i}}+\beta_{7}{ }^{*}$ Prop_5_Accionistas $_{i}+\beta_{8}{ }^{*} \mathrm{~N} 1 \mathrm{AF}_{\mathrm{i}}+\beta_{9}{ }^{*} \mathrm{~N} 1{ }_{-} \mathrm{PF} \mathrm{F}_{\mathrm{i}}+\beta_{10}{ }^{*} \mathrm{~N} 2{ }_{-} \mathrm{AF}_{\mathrm{i}}+\beta_{11}{ }^{*} \mathrm{~N} 2{ }_{-} \mathrm{PF}_{\mathrm{i}}+$ $\beta_{12}{ }^{*} \mathrm{~N} 3 \_\mathrm{AF}_{\mathrm{i}}+\beta_{13}{ }^{*} \mathrm{~N} 3 \mathrm{PF}_{\mathrm{i}}+\beta_{14}{ }^{*} \mathrm{DIS}_{\mathrm{i}}+\beta_{15}{ }^{*}$ Botosan $_{\mathrm{i}}+\mu_{\mathrm{i}}$

(ecuación 5)

\section{RESULTADOS}

Respecto a los modelos donde se utiliza la variable dicotómica $V R$ para modelar la adopción o no de las disposiciones IFRS 13, en los resultados presentados en las Tablas II y III es posible apreciar niveles de significatividad individual y conjunta para las variables del modelo del $99 \%$. La variabilidad de la variable endógena es explicada, en un $64 \%$ por la variabilidad de las variables exógenas, para los modelos de las tablas II y III.

Tabla II. Resultados estimación modelo ecuación 2.

\begin{tabular}{|l|l|l|l|l|l|l|}
\hline \multirow{2}{*}{ Variables } & \multicolumn{2}{|l|}{ Coeficientes no estandarizados } & \multirow{2}{*}{} & & \multicolumn{2}{|l|}{ Estadísticos de colinealidad } \\
\cline { 2 - 3 } \cline { 6 - 7 } & $\mathrm{B}$ & Error típ. & Estadístico $\mathrm{t}$ & Sig. & Tolerancia & FIV \\
\hline (Constante) & 0.08003746 & 0.008009073 & 9.993348884 & $1.86839 \mathrm{E}-19$ & & \\
P_Bursatil & -0.0004169 & $3.7327 \mathrm{E}-05$ & -11.1688708 & $5.38537 \mathrm{E}-23$ & 0.662682925 & 1.509017302 \\
IFRS & -0.00701975 & 0.001620278 & -4.33243185 & $2.29426 \mathrm{E}-05$ & 0.945864488 & 1.057233898 \\
IPSA & -0.00474931 & 0.001435445 & -3.30859973 & 0.001104888 & 0.570060863 & 1.754198656 \\
Ln_Activos & -0.00093348 & 0.000386087 & -2.41780357 & 0.016476226 & 0.743284208 & 1.345380393 \\
\hline
\end{tabular}

Fuente: Elaboración propia.

Tabla III. Resultados estimación modelo ecuación 3.

\begin{tabular}{|c|c|c|c|c|c|c|}
\hline \multirow{2}{*}{ Variables } & \multicolumn{2}{|c|}{ Coeficientes no estandarizados } & \multirow[b]{2}{*}{ Estadístico $t$} & \multirow[b]{2}{*}{ Sig. } & \multicolumn{2}{|c|}{ Estadísticos de colinealidad } \\
\hline & $\mathrm{B}$ & Error típ. & & & Tolerancia & FIV \\
\hline (Constante) & 0.077568338 & 0.007389018 & 10.49778779 & $5.83561 \mathrm{E}-21$ & & \\
\hline P_Bursatil & -0.0004095 & 3.44372E-05 & -11.8912047 & $3.22656 \mathrm{E}-25$ & 0.662682925 & 1.509017302 \\
\hline IFRS & -0.00637056 & 0.001494838 & -4.26170714 & 3.07478E-05 & 0.945864488 & 1.057233898 \\
\hline IPSA & -0.00455556 & 0.001324314 & -3.43993736 & 0.000702994 & 0.570060863 & 1.754198656 \\
\hline Ln_activos & -0.00088966 & 0.000356197 & -2.49765194 & 0.013276972 & 0.743284208 & 1.345380393 \\
\hline
\end{tabular}

Fuente: Elaboración propia.

Al observar los resultados de la Tabla IV y Tabla V se puede deducir que las variables que aportan significativamente a la explicación de la asimetría de información son: P_Bursatil, IFRS, IPSA, y LN_Activos; los resultados en términos de su significatividad estadística son indiferen-

9 Valor- $\mathrm{p}=<0,0164$, Estadístico $\mathrm{t}=<-2,4178$; Estadístico F Tabla $3=87,067$; Estadístico F Tabla 4=96,406 
tes según la forma de calcular el spread. Los signos de las variables exógenas son coincidentes con la literatura revisada. Así, cuando aumentan los niveles de presencia bursátil ( $P \_$Bursatil) disminuye la asimetría de información (Spread). Esto ayuda a sostener que, los inversionistas pueden percibir menor asimetría de información cuando la liquidez bursátil del título de la compañía es mayor. Además, en la medida que es mayor el tamaño de las empresas ( $L n_{-}$Activos) menor es la asimetría de información. De igual manera, y coincidiendo con las evidencias de la literatura revisada, cuando las compañías participan en el IPSA la asimetría de información es menor.

Respecto a la variable que mide el efecto de la adopción de las normas internacionales de información financiera por las empresas de la muestra (IFRS) resulta ser significativa e inversamente proporcional al spread: cuando las empresas han adoptado la normativa internacional presentan menor asimetría de información (spread) que cuando no lo han hecho. Esto puede deberse a que los inversionistas perciben menor incertidumbre por la mayor revelación de información (cuando adoptan las IFRS se basa principalmente sobre valores económicos y no sobre valores históricos), lo que generará menor asimetría de información para los inversionistas (menor spread). Respecto a la variable vinculada a la aplicación anticipada de la IFRS 13 por parte de las empresas $(V R)$, esta variable no resulta ser significativa en los modelos planteados.

Ahora bien, en relación a los modelos donde se cuantifica la medición de los activos y pasivos financieros de cada una de las empresas de la muestra distinguiendo las jerarquías de niveles según los datos de entradas que han utilizado en la técnica de valoración, en los resultados de los modelos presentados en las Tablas IV y V, se pueden apreciar niveles de significatividad individual y conjunta para las variables del modelo del $99 \%{ }^{10}$. La variabilidad de la variable endógena es explicada, en un $70 \%$, por la variabilidad de las variables exógenas, para los modelos de las Tablas IV y V.

Tabla IV. Resultados estimación modelo ecuación 4.

\begin{tabular}{|c|c|c|c|c|c|c|}
\hline \multirow{2}{*}{ Variables } & \multicolumn{2}{|c|}{ Coeficientes no estandarizados } & \multirow[b]{2}{*}{ Estadístico $t$} & \multirow[b]{2}{*}{ Sig. } & \multicolumn{2}{|c|}{ Estadísticos de colinealidad } \\
\hline & B & Error típ. & & & Tolerancia & FIV \\
\hline (Constante) & 0.083943376 & 0.008478413 & 9.900835381 & $8.14266 \mathrm{E}-16$ & & \\
\hline P_Bursatil & -0.00036906 & 4.45914E-05 & -8.27644564 & $1.57008 \mathrm{E}-12$ & 0.582909613 & 1.715531837 \\
\hline Botosan & -0.00023572 & $6.36108 \mathrm{E}-05$ & -3.70572808 & 0.000374351 & 0.940619171 & 1.063129511 \\
\hline Ln_activos & -0.00147537 & 0.000418983 & -3.5213073 & 0.000693191 & 0.630987354 & 1.584817815 \\
\hline N1_AF & 0.035870387 & 0.017711001 & 2.025316693 & 0.045972439 & 0.503245615 & 1.987101267 \\
\hline
\end{tabular}

Fuente: Elaboración propia.

Tabla V. Resultados estimación modelo ecuación 5.

\begin{tabular}{|l|l|l|l|l|l|l|}
\hline \multirow{2}{*}{ Variable } & \multicolumn{2}{|l|}{ Coeficientes no estandarizados } & & & \multicolumn{2}{|l|}{ Estadísticos de colinealidad } \\
\cline { 2 - 5 } \cline { 6 - 8 } & B & Error típ. & Estadístico t & Sig. & Tolerancia & FIV \\
\hline (Constante) & 0.083841144 & 0.008404832 & 9.975350675 & $5.76185 \mathrm{E}-16$ & & \\
P_Bursatil & -0.00036857 & $4.42044 \mathrm{E}-05$ & -8.33797719 & $1.17954 \mathrm{E}-12$ & 0.582909613 & 1.715531837 \\
Botosan & -0.00023522 & $6.30587 \mathrm{E}-05$ & -3.73012536 & 0.00034455 & 0.940619171 & 1.063129511 \\
Ln_activos & -0.00147327 & 0.000415347 & -3.54707318 & 0.000636769 & 0.630987354 & 1.584817815 \\
N1_AF & 0.035387395 & 0.017557293 & 2.01553827 & 0.047007475 & 0.503245615 & 1.987101267 \\
\hline
\end{tabular}

Fuente: Elaboración propia. 
Al observar los resultados de la Tabla IV y Tabla $\mathrm{V}$ se puede deducir que las variables que aportan significativamente a la explicación de la asimetría de información son: $P \_$Bursatil, Botosan, Ln_Activos, N1_AF; los resultados en términos de su significatividad estadística son indiferentes según la forma de medir el spread. Los signos de las variables exógenas son coincidentes con la literatura revisada. Así, y al igual que en los anteriores modelos, cuando aumentan los niveles de presencia bursátil (P_Bursatil) disminuye la asimetría de información (Spread). Cabe destacar que finalmente en los modelos presentados se utiliza como medida de calidad de los gobiernos corporativos el Botosan y no el DIS, dado que ambas variables presentan una significativa medida de correlación ${ }^{11}$. Respecto a la variable que mide la calidad de los gobiernos corporativos (Botosan), a mejor calidad de gobiernos en las empresas la asimetría de información disminuye. A mayor tamaño de las empresas de la muestra ( $L n_{-}$Activos) disminuye la asimetría de información. Por último, y respecto a las tres medidas de valoración para los activos y pasivos financieros, podemos advertir que mientras más objetiva es la valoración del activo financiero (N1_AF), se percibe mayor asimetría de información, esto debido a que se utilizan precios cotizados en un mercado más activo que para el caso de un nivel mayor de valoración (ejemplo, N2_AF) en que se usan los precios cotizados en mercados de referencia o en mercados no activos.

\section{CONCLUSIONES}

En la presente investigación se ha estudiado el efecto que tienen la calidad de gobiernos corporativos medida a través de un índice de revelación (Botosan) y el uso del valor razonable, sobre la asimetría de información percibida por los inversionistas en el mercado de capitales chileno. Así, a través del estudio se ha logrado: (i) construir y analizar modelos que interpretan la relación que se produce entre el índice de revelación de la calidad de los gobiernos corporativos y la asimetría de información (Spread); (ii) analizar la relación que existe entre la asimetría de información, medida por el spread, y las medidas de valoración (Nivel 1, Nivel 2 y Nivel 3) implementadas bajo la aplicación de IFRS 13. También se ha logrado estudiar el efecto que tienen en la asimetría de información (a) la adopción anticipada de las IFRS 13 en Chile, y, (b) el efecto de la adopción de las IFRS, de la participación en el IPSA, de la presencia bursátil y del total de activos, de las empresas de la muestra. A pesar que se ha logrado demostrar que para las empresas estudiadas, a mayor calidad de gobiernos corporativos medida por el índice Botosan, disminuye la asimetría de información, medida por el spread, se ha tenido que rechazar la hipótesis que plantea que la aplicación anticipada de la IFRS 13 genera menos asimetría de información. Una explicación plausible de que la aplicación anticipada de la IFRS 13 no ha significado una disminución en la asimetría de información en Chile es que los inversionistas aún no han asimilado la forma en que las compañías miden y revelan el valor razonable de sus activos y pasivos financieros, o bien no es suficientemente relevante y confiable para los inversionistas como para reflejarse en los precios de las acciones (Song, Thomas \& Yi, 2010).

La asimetría de información sólo se reducirá en la medida que los inversionistas observen menor incertidumbre en la información que les proveen. Por otra parte, y al separar en tres los niveles de valoración para los activos y pasivos financieros, la evidencia empírica con los datos de esta muestra estaría indicando que el modelo de valoración en base a valor razonable no ha contribuido a disminuir la asimetría de información como se esperaba. La literatura revisada anteriormente avala esta conclusión, pues no hay estudios concluyentes sobre esta materia.

Como futuros trabajos se pretende ampliar el tamaño de la muestra en términos del número de empresas así como en los periodos a considerar, objeto observar si las conclusiones a las que se ha llegado en esta investigación se mantienen dado el mayor periodo de la aplicación obligatoria de la norma.

\section{REFERENCIAS}

Armstrong, C. S., Barth, M. E., Jagolinger, A. D. \& Riedl, E. J. (2010). Market Reaction to the Adoption of IFRS in Europe. The Accounting Review, 85(1), 31-61.

Assidi, S. \& Omri, M. A. (2012). IFRS and Infor-

11 Coeficiente de correlación 0,482473; Valor-p: 0,0000 
mation Quality: Cases of CAC 40 Companies. Global. Journal of Management and Business Research, 12(19), 72-83.

Bakhshi, M., Bazrafshan, E., Rezaei, M. \& Fereidouni, H.G. (2011). The Impact of Corporate Governance on the Bid-Ask Spread: Evidence from an Emerging Market. International Conference on Sociality and Economics Development IPEDR, volumen 10, IACSIT Press, Singapore. Recuperado de http://www.ipedr.com/ vol10/97-S10064.pdf

Barth, M. \& Landsman, W. (2010). How did financial reporting contribute to the Financial Crisis? European Accounting Review, 19(3), 399423.

Barth, M. Landsman, W., Lang, M. \& Williams, C. (2012). Are IFRS-based and US GAAP-based Accounting Amounts Comparable? Journal of Accounting \& Economics, 54(1), 68-93.

Botosan, C. A. (1997). Disclosure Level and The Cost of Equity Capital. The Accounting Review, 72(3), 323-349.

Chen, W. P., Chung, H., Lee, C. \& Liao, W.L. (2007). Corporate Governance and Equity Liquidity: analysis of S\&P transparency and disclosure rankings. Corporate Governance: An International Review, 15(4), 644-660.

Clarkson, P., Hanna, J., Richardson, G. \& Thompson, R. (2011). The impact of IFRS adoption on the value relevance of book value and earnings. Journal of Contemporary Accounting and Economics, 7(1), 1-17.

Copeland, T. E. \& Galai, D. (1983). Information Effects on the Bid-Ask Spread. The Journal of Finance, 38(5), 1457-1469.

De la Fuente, H.; Campos, R.; Silva, B. \& Cademartori, D. (2013). "An econometric analysis for the behavior of the bid-ask spread". Open Journal of Social Sciences, vol. 1(7), pp. 1-5.

Dennis, P. \& Weston, J. (2001). Who's Informed? An Analysis of Stock Ownership and Informed Trading (June 4, 2001). AFA 2002 Atlanta Meetings. Available at SSRN: http://ssrn.com/abstract $=267350$ or http://dx.doi.org/10.2139/ $\underline{\text { ssrn. } 267350}$
Erwin, G. R. \& J. M. Miller (1998). The liquidity effects associated with addition of a stock to the S\&P 500 index: evidence from bid/ask spreads. The Financial Review. 33, 131-146

Florou, A. \& Pope, P. (2012). Mandatory IFRS Adoption and Institutional Investment Decisions. The Accounting Review, 87(6), 1993-2025.

Gjerde, T., Mahenthiran, S. \&Cademartori, D. (2013). Effect of Ownership, Governance, and Transparency on Liquidity-Chilean Evidence. Journal of Contemporary Accounting \& Economics, 9(2), 183-202.

Glosten, L. R. \& Harrris, L. E. (1988). Estimating the Components of the BID/ASK Spread. Journal of Financial Economics, 21(1), 123-142.

Haat, H. M., Mahenthiran, S., Rahman A. R. \& Hamid A. N. (2006). Agency Costs as a Factor in the Suspension of Companies from the Kuala Lumpur Stock Exchange, Journal of Contemporary Accounting and Economics, 2(1), 99-121.

He, X. Wong, T.J. \& Young, D. (2012). Challenges for implementation of fair value accounting in emerging markets: evidence from China. Contemporary Accounting Research, 29(2), 538-562.

Healy, P. M. \& Palepu, K. G. (2001). Information asymmetry, corporate disclosure, and the capital markets: A review of the empirical disclosure literature. Journal of Accounting and Economics, 31(1-3), 405-440.

International Accounting Standards Board IASB (2011). Norma Internacional de Información Financiera 13. Medición del Valor Razonable.

Kanagaretnam, K., Lobo, G. \& Whalen, D. (2007). Does good corporate governance reduce information asymmetry around quarterly earnings announcements? Journal of Accounting and Public Policy, 26(4), 497-522.

Kim, O \& Verrecchia, R. (1994). Market liquidity and volumen around earnings announcements. Journal of Accounting and Economics, 17, 41-67.

Liao, Kang, Morris \& Tang. (2013). Information asymmetry of fair value accounting during the financial crisis. Journal of Contemporary Accounting \& Economics, 9(2), 221-236. 
Maug, E.G. (2002). Insider Trading Legislation and Corporate Governance. European Economic Review, 46(9), 1569-1597.

Munteanu, L. (2011). Cost of Equity, Financial Information Disclosure, and IFRS adoption: A Literature Review. Internal Auditing \& Risk Management, 4(24), 67-80.

Muller, K., Riedl, E. \& Sellhorn, T. (2011). Mandatory Fair Value Accounting and Information Asymmetry: Evidence from the European Real Estate Industry. Management Science, 57(6), 1138-1153.

Organización para la Cooperación y el Desarrollo Económico (OCDE). (2011). Fortaleciendo el Gobierno Corporativo Latinoamericano. El papel de los inversionistas institucionales, 1-80.

Rubin, A. (2007). Ownership Level, Ownership Concentration and Liquidity. Journal of Financial Markets, 10(3), 219-248.

Sarin, A., Shastri, K. \& Shastri, K. (1996). Ownership structure and stock market liquidity. Disponible en SSRN: http://ssrn.com/abstract $=2652$

Song, C. J., Thomas W. B. \&Yi, H. (2010) Value Relevance of FAS No. 157 Fair Value Hierarchy Information and the Impact of Corporate Governance Mechanisms. The Accounting Review, 85(4), 1375-1410.

Venkatesh, P.C. \& Chiang, R. (1986). Information Asymmetry and the Dealer's Bid-Ask Spread: A Case Study of Earnings and Dividend Announcements. Journal of Finance, 41(5), 10891102. 